\title{
SPACES OF RIEMANN SURFACES AS BOUNDED DOMAINS ${ }^{1}$
}

\author{
BY LIPMAN BERS
}

Communicated November 14, 1959

1. A surface of type $(g, n)$ is a surface $S$ obtained by removing $n \geqq 0$ distinct points from a closed Riemann surface of genus $g$; we assume always that $3 g-3+n>0$. Such a surface $S$ is marked by choosing a homotopy class $[f]$ of orientation preserving homeomorphisms $f$ of $S$ onto a fixed reference surface $S_{0}$. Two marked surfaces $\left(S_{1},\left[f_{1}\right]\right)$ and $\left(S_{2},\left[f_{2}\right]\right)$ are equivalent if there is a conformal mapping $g$ with $g\left(S_{1}\right)=S_{2},\left[f_{2} g\right]=\left[f_{1}\right]$. The Teichmüller space $T_{g, n}$ is the set of equivalence classes of marked surfaces of type $(g, n)$. It is known that $T_{g, n}$ carries two natural structures: that of a metric space, homeomorphic to a $(6 g-6+2 n)$-cell $[1 ; 4 ; 10 ; 11]$, and that of a complex manifold $[2 ; 5 ; 7 ; 8 ; 12]$.

We sketch in $\$ \S 2-7$ a proof of the

THEOREM. $T_{g, n}$ is (holomorphically equivalent to) a bounded domain in complex number space.

In $\$ \S 8-10$ we consider factor spaces of $T_{\imath, n}$.

2. A group $G$ of Möbius transformations is called a Schottky group of genus $g$ if there exists a closed region $R$ (standard fundamental region) on the Riemann sphere $P$, bounded by $2 g$ disjoint Jordan curves $C_{j}$, and $g$ elements $A_{1}, \cdots, A_{g}$ of $G$ (standard set of generators) with $A_{j}\left(C_{2 j-1}\right)=C_{2 j}$. In this case the $A_{j}$ generate $G$, the limit points of $G$ form a perfect nowhere dense set $Q(G)$ of measure zero, $G$ is properly discontinuous and fixed-point-free on $P-Q(G)$, and $(P-Q(g)) / G$ is a closed Riemann surface of genus $g$.

Every closed Riemann surface can be so represented.

This classical theorem (Klein-Koebe-Courant) can be proved as follows. Let $G_{0}$ be a fixed Schottky group and $S$ a given closed Riemann surface of the same genus. Then $S$ can be represented as the surface $\left(P-Q\left(G_{0}\right)\right) / G_{0}$ on which the conformal structure has been redefined by means of a Riemannian metric $d s=|d z+\mu(z) d \bar{z}|$, where $\mu(z)$ is measurable, $\mu(z) d z / d \bar{z}$ is invariant under $G_{0}$ and $|\mu(z)| \leqq k<1$ (cf. $[3 ; 4])$. Let $z \rightarrow W(z)$ be the uniquely determined homeomorphism

1 This paper represents results obtained at the Institute of Mathematical Sciences, New York University, under the sponsorship of the Office of Ordnance Research, U. S. Army, Contract No. DA-30-069-ORD-2153. Reproduction in whole or in part permitted for any purpose of the United States Government. 
of $P$ onto itself which leaves $0,1, \infty$ fixed and is conformal with respect to $d s$. Then the mapping $z \rightarrow W(A(z)), A \in G_{0}$, is also conformal with respect to $d s$, so that $W(A(z))=B(W(z))$ where $B$ is a Möbius transformation. One verifies that $G=W G_{0} W^{-1}$ is a Schottky group and $S=(P-Q(G)) / G$.

Two properties of $W$ are crucial for what follows. (I) If $\mu(z) \mathrm{de}-$ pends continuously, in the sense of bounded a.e. convergence, on real parameters, then $W(z)$ depends continuously on these parameters. (II) If $\mu(z)$ is a holomorphic function of complex parameters, so is $W(z)$ (cf. $[3]$ ).

3. We denote by $\Delta$ the domain $1<|\lambda|<\infty$ and by $L_{k}, k>3$, the complex manifold of points $\zeta=\left(\zeta_{1}, \zeta_{2}, \cdots, \zeta_{k-3}\right)$ with $\zeta_{j} \neq 0,1, \infty$ and $\zeta_{i} \neq \zeta_{j}$ for $i<j$. We define the set

$$
\Sigma_{g, n} \subset L_{2 g+n} \times \Delta^{g}
$$$$
(g>0,3 g-3+n>0)
$$

and the set $\Theta_{g, n} \subset \Sigma_{g, n}$ by the following conditions. For $\zeta=\left(\zeta_{1}, \cdots, \zeta_{2 g+n-3}\right) \in L_{2 g+n}$ set $\sigma_{1}=0, \sigma_{1}^{\prime}=\infty, \sigma_{2}=1, \sigma_{2}^{\prime}=\zeta_{1}, \sigma_{3}$ $=\zeta_{2}, \cdots, \sigma_{g}^{\prime}=\zeta_{2 g-3}, p_{i}=\zeta_{2 g-2}, \cdots, p_{n}=\zeta_{2 g+n-3}$ if $g>1$ and $\sigma_{1}=0$, $\sigma_{1}^{\prime}=\infty, p_{1}=1, p_{2}=\zeta_{1}, \cdots, p_{n}=\zeta_{n-1}$ if $g=1$. For $\lambda=\left(\lambda_{1}, \cdots, \lambda_{g}\right)$ $\in \Delta^{o}$ let $A_{j}$ be the Möbius transformation with repelling fixed point $\sigma_{j}$, attracting fixed point $\sigma_{j}^{\prime}$, and with multiplier $\lambda_{j}$. Then $(\zeta, \lambda) \in \Sigma_{o, n}$ if $\left(A_{1}, \cdots, A_{g}\right)$ is a standard set of generators of a Schottky group $G$ with a standard fundamental region $R$ containing all points $p_{1}, \cdots, p_{n}$ in its interior, and $(\zeta, \lambda) \in \Theta_{g, n}$ if the boundary curves $C_{2 j-1}, C_{2 j}$ of $R$ can be chosen as Apollonius circles with respect to the pairs $\left(\sigma_{j}, \sigma_{j}^{\prime}\right), j=1, \cdots, g$.

We define the set

$$
\mathrm{H}_{g} \subset L_{g+2} \times \Delta^{g}
$$

by the following condition. For $\zeta \in L_{g+2}, \lambda \in \Delta^{g}$ set $\tau_{1}=1, \tau_{j}=\zeta_{j-1}$, $j=2, \cdots, g$, and let $A_{j}$ be a Möbius transformation with fixed points $\pm \tau_{j}^{1 / 2}$ and multiplier $\lambda_{j}$. Then $(\zeta, \lambda) \in \mathrm{H}_{g}$ if $\left(A_{1}, \cdots, A_{g}\right)$ is a standard set of generators for a Schottky group $G$ with a standard fundamental region $R$ which is invariant under the mapping $z \rightarrow-z$.

We define the set

$$
\Xi_{2 g} \subset L_{g+3} \times \Delta^{g}
$$

by the following condition. For $\zeta \in L_{g+3}, \lambda \in \Delta^{\theta}$ let $\sigma_{j}$ be a root of the equation $\sigma_{j}^{2}+\sigma_{j}^{-2}-2=2 \zeta_{j}$, and let $A_{j}$ and $A_{g+j}$ be Möbius transformations with multiplier $\lambda_{j}$ and with fixed points $\pm \sigma_{j}^{1 / 2}$ and $\pm \sigma_{j}^{-1 / 2}$, respectively. Then $(\zeta, \lambda) \in \Xi_{2 g}$ if $\left(A_{1}, \cdots, A_{2 g}\right)$ is a standard 
set of generators of a Schottky group $G$ with a standard fundamental region $R$ which is invariant under the mappings $z \rightarrow-z$ and $z \rightarrow 1 / z$.

4. If $X$ and $Y$ are complex manifolds, we write $X=\tilde{Y}$ whenever $X$ is (holomorphically equivalent to) the universal covering of $Y$. In particular

$$
\tilde{\Delta}=\tilde{L}_{4}=D
$$

where $D$ is the unit disc.

Lemma A. The sets $L_{n}, \Sigma_{\theta, n}, \mathrm{H}_{g}$ and $\Xi_{\theta}$ are domains and

$$
\tilde{L}_{n}=T_{0, n}, \quad \tilde{\Sigma}_{g, n}=T_{g, n}, \quad \tilde{\mathrm{H}}_{g}=T_{0,2 g+2}, \quad \tilde{\Xi}_{2 \theta}=T_{0,2 g+3} .
$$

This can be proved by the method described in $\$ 2$, by making use of the following observations. (1) Every point of $L_{n}$ represents a surface of type $(0, n)$. (2) Every point of $\Sigma_{g, n}$ represents a surface of type $(g, n)$, the "removed" points being the points $p_{1}, \cdots, p_{n}$. (3) Every point of $\mathrm{H}_{g}$ represents a closed Riemann surface of genus $g$ which admits a conformal involution with $2 g+2$ fixed points. Such a surface is hyperelliptic; it belongs to an algebraic equation of the form

$$
w^{2}=z(z-1)\left(z-\alpha_{1}\right) \cdots\left(z-\alpha_{2 q-1}\right)
$$

with $\left(\alpha_{1}, \cdots, \alpha_{2 g-1}\right) \in L_{2 g+2}$. (4) Every element of $\Xi_{2 g}$ represents a closed Riemann surface of genus $2 g$ which admits 3 commuting conformal involutions with $4 g+2,2$ and 2 fixed points, respectively. Such a surface belongs to an algebraic equation of the form

$$
w^{2}=\left(z^{2}-1\right)\left(z^{2}-\alpha_{1}\right) \cdots\left(z^{2}-\alpha_{2 \theta}\right)
$$

with $\left(\alpha_{1}, \cdots, \alpha_{2 \theta}\right) \in L_{2 \theta+3}$.

5. Let $X$ and $Y$ be complex manifolds. An inclusion $X \subset Y$ will be called sharp if the homomorphism of fundamental groups induced by it is an isomorphism onto.

Lemma B. The inclusions (1), (2), (3) are sharp.

We sketch the proof for the case (1). It is based on the following statements. (i) If $\zeta \in L_{2 q+n}$, then there is a $q>1$ such that if $\lambda=\left(\lambda_{1}, \cdots, \lambda_{g}\right)$ and $\left|\lambda_{j}\right|>q, j=1, \cdots, g$, then $(\zeta, \lambda) \in \Theta_{g, n}$. (ii) If $(\zeta, \lambda) \in \Theta_{g, n}$ and $\left|\lambda_{j}^{\prime}\right| \geqq\left|\lambda_{j}\right|, j=1, \cdots, g$, then $\left(\zeta, \lambda^{\prime}\right) \in \Theta_{g, n}$ (iii) $\Theta_{g, n}$ is a domain. (iv) The inclusion $\Theta_{g, n} \subset L_{2 n+g} \times \Delta^{o}$ is sharp. (v) Every closed curve in $\Sigma_{\theta, n}$ beginning in $\Theta_{\theta, n}$ is homotopic to a curve in $\Theta_{\theta, n}$. 
The proofs of (i) and (ii) are elementary; these statements imply easily (iii) and (iv). The proof of (v) involves an explicit construction described below. The sharpness of (1) follows at once from (iv) and (v).

6. We prove now assertion ( $v$ ) of $\$ 6$ assuming, for the sake of brevity, that $g>1, n=0$. Without loss of generality we may assume that the given curve $t \rightarrow(\zeta(t), \lambda(t)) \in T_{g, 0}, 0 \leqq t \leqq 1$, is of class $C_{\infty}$; by hypothesis $(\zeta(0), \lambda(0))=(\zeta(1), \lambda(1)) \in \Theta_{g, 0}$. For each $t$ we can find a standard fundamental region $R^{0}(t)$ belonging to $(\zeta(t), \lambda(t))$ such that the $2 g$ boundary curves $C_{j}^{0}(t)$ of $R^{0}(t)$ are real analytic and depend on $t$ in a $C_{\infty}$ manner, and such that $C_{2 j-1}^{0}(0)=C_{2 j-1}^{0}(1), C_{2 j}(0)=C_{2 j}(1)$ are Apollonius circles with respect to $\sigma_{j}(1), \sigma_{j}^{\prime}(1)$. If $q>0$ is a large constant and $q=(q, \cdots, q)$, then $(\zeta(t), q) \in \Theta_{g}$ and we can find for each $t$ a standard fundamental region $R^{1}(t)$ belonging to $(\zeta(t), q)$ and bounded by Apollonius circles $C_{j}^{1}(t)$ such that: $C_{j}^{1}(t)$ depends in a $C_{\infty}$ way on $t, C_{2}^{1}(t)$ lies in the exterior of $C_{2}^{0}(t), C_{j}(t)$ lies in the interior of $C_{j}^{0}(t), j \neq 2$. Let $\phi_{j, t}$ be the conformal mapping of the domain bounded by $C_{j}^{0}(t)$ and $C_{j}^{1}(t)$ onto an annulus $0>r_{j}(t)>|w|>1$. We can normalize $\phi_{j, t}$ so that it maps $C_{j}^{0}(t)$ onto $|w|=r_{j}(t)$ and is a $C_{\infty}$ function of $t$.

For fixed $t$ and $s, 0 \leqq t \leqq 1,0 \leqq s \leqq 1$, let $K_{j}(t, s)$ be the $\phi_{j, t}^{-1}$-image of the circle $|w|=r_{j}(t)+s\left[1-r_{j}(t)\right]$. The curves $K_{j}(t, s)$ bound a closed region $M(t, s)$ of connectivity $2 g$. The Möbius transformations determined by $(\zeta(t), \lambda(t))$ and by $(\zeta(t), q)$ define real analytic homeomorphisms of $C_{2 j-1}^{0}(t)$ onto $C_{2 j}^{0}(t)$ and of $C_{2 j-1}^{1}(t)$ onto $C_{2 j}^{1}(t)$, respectively. We "interpolate" between these, using the functions $\phi_{j, t}$, and obtain real analytic homeomorphisms $\omega_{j, t, s}$ of $K_{2 j-1}(t, s)$ onto $K_{2 j}(t, s)$. Identifying boundary points of $M(t, s)$ corresponding to each other under $\omega_{j, t, s}$ we obtain a closed Riemann surface $S$ of genus $g$. There exists a unique point $(\zeta(t, s), \lambda(t, s)) \in T_{g, 0}$ such that the Schottky group defined by it represents $S$ and has a standard fundamental region bounded by images of the curves $K_{j}(t, s)$, in the "correct" order.

The mapping $(t, s) \rightarrow(\zeta(t, s), \lambda(t, s))$, of the unit square into $T_{g, 0}$, just constructed can be shown to be continuous. On the edge $s=0$, it coincides with the given curve; the other three edges are mapped into $\Theta_{g, 0}$. Thus the given curve is homotopic to a curve in $\Theta_{0,0}$.

7. If $X \subset Y$ is a sharp inclusion between connected complex manifolds, then it is easy to construct (canonically) an embedding $\tilde{X} \subset \tilde{Y}$. This remark, together with (4) and Lemmas A and B, yield the relations 


$$
\begin{aligned}
T_{g, n} & \subset T_{0,2 g+n} \times D^{g}, \\
T_{0,2 g+2} & \subset T_{0, g+2} \times D^{g}, \\
T_{0,2 g+3} & \subset T_{0, g+3} \times D^{g} .
\end{aligned}
$$

By induction we obtain from (4), (6) and (7) that

$$
T_{0, n} \subset D^{n-3},
$$

Thus, by (5),

$$
T_{g, n} \subset D^{3 g-3 n+n} \quad(g>0, n \geqq 0,3 g-3+n>0) .
$$

The inclusions (8), (9) contain our theorem.

8. Let $S_{0}$ be the reference surface of type $(g, n)$ and $\gamma_{g, n}$ its fundamental group. Let $\Lambda_{\theta, n}$ denote the group of homotopy classes [ $\left.\phi\right]$ of orientation-preserving homeomorphisms of $S_{0}$ onto itself; this group is canonically identified with a subgroup of the group of automorphisms of $\gamma_{g, n}$, modulo inner automorphisms. Every $[\phi] \in \Lambda_{g, n}$ induces a self-mapping $p[\phi]$ of $T_{g, n}$ which sends the equivalence class of $(S,[f])$ into that of $(S,[\phi f])$. We denote by $\Gamma_{o, n}$ the image of $\Lambda_{g, n}$ under the homomorphism $p$. For $g+n>2 p$ is an isomorphism.

The group $\Gamma_{a, n}$ is a properly discontinuous group of holomorphic automorphisms. Hence, by a theorem of H. Cartan [6] every subgroup $K \subset \Gamma_{\theta, n}$ gives rise to a normal complex space $T_{g, n} / K$. In particular, $R_{\theta, n}=T_{g, n} / \Gamma_{g, n}$, i.e. the space of conformal equivalence classes of surfaces of type $(g, n)$ is a normal complex space. (A quite different proof of this for $n=0$ is due to Röhrl [9].)

9. For $n>0$ let $S_{0}^{*}$ denote the (uniquely determined) closed surface containing $S_{0}$. We say that $[\phi] \in \Gamma_{\theta, n}^{*}$ if $[\phi] \in \Gamma_{\theta, n}$ and $\phi$ is homotopic to the identity as a mapping of $S_{0}^{*} . T_{g, n} / \Gamma_{g, n}^{*}$ is a complex manifold, for $\Gamma_{0, n}^{*}$ is fixed point free.

Of particular interest is $T_{\theta}^{(1)}=T_{g, 1} / \Gamma_{g, 1}^{*}$. There is an obviously defined holomorphic mapping $\varpi$ of $T_{0}^{(1)}$ onto $T_{o, 0}$, if $t$, the equivalence class of $(S,[f])$, is a point of $T_{g, 0}$, then $\varpi^{-1}(t)$ is a Riemann surface conformally equivalent to $S$ and regularly embedded in $T_{\theta}^{(1)}$ (cf. [5]).

10. Let $g>1$ and let $a_{1}, b_{1}, \cdots, b_{g}, c_{1}, \cdots, c_{n}$ be a "standard" set of generators for $\gamma_{\theta, n}$ (the $a$ 's and $b$ 's come from a "canonical dissection" of $S_{0}^{*}$ and $c_{j}$ comes from the $j$ th distinguished point). Let $\delta_{g, n}$ denote the smallest normal subgroup containing all $b$ 's and $c$ 's, $h_{g, n}$ the group of automorphisms of $\gamma_{g, n}$ which belong to $\Lambda_{g, n}$, leave $\delta_{g, n}$ fixed, and induce an inner automorphism on $\gamma_{g, n} / \delta_{g, n}$, and let $H_{g, n}=p h_{g, n}$ be the "corresponding" subgroup of $\Gamma_{g, n}$. 
One can easily describe $h_{\theta, n}$ once $H_{\theta, n}$ is known. On the other hand, one can show that $T_{g, n} / H_{g, n}=\Sigma_{g, n}$ so that $H_{g, n}$ is canonically isomorphic to the fundamental group of $\Sigma_{g, n}$. Thus Lemma B contains a purely algebraic result which seems to be difficult to obtain by purely algebraic (or topological) methods.

\section{REFERENCES}

1. L. V. Ahlfors, On quasi-conformal mappings, J. Analyse Math. vol. 3 (19531954) pp. 1-58.

2. - The complex analytic structure of the space of closed Riemann surfaces, to appear.

3. L. V. Ahlfors and Lipman Bers, Riemann's mapping theorem for variable metrics, to appear.

4. Lipman Bers, Quasiconformal mappings and Teichmilller's theorem, to appear.

5. - Spaces of Riemann surfaces, Proceedings of the International Congress of Mathematicians, Edinburgh, 1958, pp. 349-361.

6. Henri Cartan, Quotient d'un espace analytique par un groupe d'automorphismes, Algebraic Geometry and Topology, A Symposium in honor of S. Lefschetz, Princeton University Press, 1957, pp. 90-102.

7. Kunihiko Kodaira and D. C. Spencer, Existence of complex structure on a differentiable family of deformations of compact complex manifolds, to appear.

8. H. E. Rauch, On the transcendental moduli of algebraic Riemann surfaces, Proc. Nat. Acad. Sci. U.S.A. vol. 41 (1955) pp. 42-59.

9. Helmut Röhrl, to appear.

10. Oswald Teichmüller, Extremale quasikonforme Abbildungen und quadratische Differentiale, Abh. Preuss. Akad. Berlin no. 22 (1940).

11. - Bestimmung der extremalen quasikonformen Abbildungen bei geschlossnen Riemannschen Flächen, Abh. Preuss. Akad. Berlin no. 4 (1943).

12. André Weil, Sur les modules des surfaces de Riemann, Séminaire Bourbaki, May, 1958.

New YoRK UNIVERSITy 